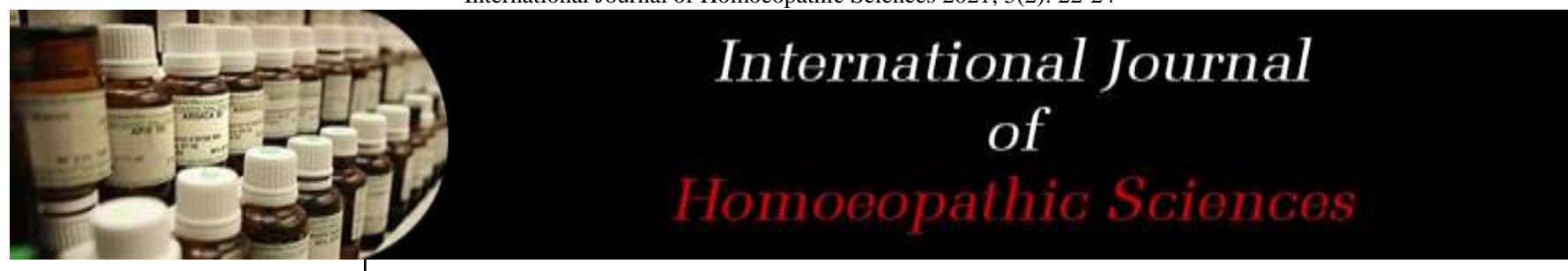

E-ISSN: 2616-4493 P-ISSN: 2616-4485 www.homoeopathicjournal.com IJHS 2021; 5(2): 22-24

Received: 17-01-2021

Accepted: 22-02-2021

Dr. Putchala Bhagya Vineesha Pg Part 1, Department of Homoeo Pharmacy, Guru Mishri Homoeopathic Medical College Shelgaon,

Maharashtra, India

Dr. Kavitha Khandare Hod, Guru Mishri

Homoeopathic Medical College Shelgaon, Maharashtra, India

Dr. Dhanshree Joshi

Pg Guide, Guru Mishri

Homoeopathic Medical College Shelgaon, Maharashtra, India

Corresponding Author: Dr. Putchala Bhagya Vineesha Pg Part 1, Department Of Homoeo Pharmacy, Guru Mishri Homoeopathic Medical College Shelgaon,

Maharashtra, India

\section{Acute nephritis and it's approach to homoeopathic therapeutics}

\author{
Dr. Putchala Bhagya Vineesha, Dr. Kavitha Khandare and Dr. \\ Dhanshree Joshi
}

DOI: $\underline{\text { https://doi.org/10.33545/26164485.2021.v5.i2a.359 }}$

\section{Abstract}

Acute nephritis occurs when your kidneys suddenly become inflamed. Acute nephritis has several causes, and it can ultimately lead to kidney failure if it's left untreated. This condition used to be known as Bright's disease. A kidney infection is a type of urinary tract infection (UTI). Your body has ways to defend against infections in the urinary tract. For example, urine normally flows one way from your kidneys to your bladder. Viruses or bacteria that enter are flushed out by urinating. This one-way flow of urine usually prevents an infection in your urinary tract. Most kidney infections are caused by bacteria or viruses, that first infect your lower urinary tract, usually your bladder. Then, the infection moves upstream to one or both of your kidneys, which are part of the upper urinary tract.

Keywords: Acute nephritis, homoeopathic therapeutics, Urinary Tract Infection

\section{Introduction}

Nephritis is the inflammation of the kidneys and may involve glomeruli, tubules, or interstitial tissue surrounding the glomeruli and tubules. Women have more bladder infections (also called urinary tract infections) than men because the distance to the bladder from skin, where bacteria normally live, is quite short and direct. However, the infection usually remains in the bladder.

\section{Types}

There are several types of acute nephritis:

\section{Interstitial nephritis}

In interstitial nephritis, the spaces between the kidney tubules become inflamed. This inflammation causes the kidneys to swell.

\section{Pyelonephritis}

Pyelonephritis is an inflammation of the kidney, usually due to a bacterial infection. In the majority of cases, the infection starts within the bladder and then migrates up the ureters and into the kidneys. Ureters are two tubes that transport urine from each kidney to the bladder.

\section{Glomerulonephritis}

This type of acute nephritis produces inflammation in the glomeruli. There are millions of capillaries within each kidney. Glomeruli are the tiny clusters of capillaries that transport blood and behave as filtering units. Damaged and inflamed glomeruli may not filter the blood properly. Learn more about glomerulonephritis.

\section{Causes}

Each type of acute nephritis has its own causes as follows:

\section{Interstitial nephritis}

This type often results from an allergic reaction to a medication or antibiotic. An allergic reaction is the body's immediate response to a foreign substance. Your doctor may have prescribed the medicine to help you, but the body views it as a harmful substance. This makes the body attack itself, resulting in inflammation. 
Low potassium in your blood is another cause of interstitial nephritis. Potassium helps regulate many functions in the body, including heartbeat and metabolism.

Taking medications for long periods of time may damage the tissues of the kidneys and lead to interstitial nephritis.

\section{Pyelonephritis}

The majority of pyelonephritis cases results from E.coli bacterial infections. This type of bacterium is primarily found in the large intestine and is excreted in your stool. The bacteria can travel up from the urethra to the bladder and kidneys, resulting in pyelonephritis.

Although bacterial infection is the leading cause of pyelonephritis, other possible causes include:

1. Urinary examinations that use a cystoscope, an instrument that looks inside the bladder

2. Surgery of the bladder, kidneys, or ureters

3. The formation of kidney stones, rocklike formations consisting of minerals and other waste material

A woman is more likely to develop pyelonephritis when she is pregnant. Pyelonephritis and other forms of urinary tract infection increase the risk of premature delivery.

A man is more likely to develop the problem if his prostate is enlarged, a common condition after age 50. Both men and women are more likely to develop pyelonephritis if they have any of the following conditions:

- An untreated urinary tract infection

- Diabetes

- Nerve problems that affect the bladder

- Kidney stones

- A bladder tumor

- Abnormal backflow of urine from the bladder to the kidneys, called vesicoureteral reflux

- An obstruction related to an abnormal development of the urinary tract

\section{Glomerulonephritis}

The main cause of this type of kidney infection is unknown. However, some conditions may encourage an infection, including:

- problems in the immune system

- a history of cancer

- an abscess that breaks and travels to your kidneys through your blood

\section{Mechanism}

Nephritis can produce glomerular injury, by disturbing the glomerular structure with inflammatory cell proliferation. This can lead to reduced glomerular blood flow, leading to reduced urine output (oliguria) and retention of waste products (uremia).As a result, red blood cells may leak out of damaged glomeruli, causing blood to appear in the urine (hematuria).

Low renal blood flow activates the renin-angiotensinaldosterone system (RAAS), causing fluid retention and mild hypertension. As the kidneys inflame, they begin to excrete needed protein from the affected individual's body into the urine stream. This condition is called proteinuria. Loss of necessary protein due to nephritis can result in several life-threatening symptoms. The most serious complication of nephritis can occur if there is significant loss of the proteins that keep blood from clotting excessively. Loss of these proteins can result in blood clots, causing sudden stroke.

\section{Clinical features}

- Pain in the pelvis

- $\quad$ pain or a burning sensation while urinating

- a frequent need to urinate

- Cloudy urine

- blood or pus in the urine

- Pain in the kidney area or abdomen

- $\quad$ swelling of the body, commonly in the face, legs, and feet

- $\quad$ vomiting

- fever

- high blood pressure

\section{Prevalence}

Nephritis represents the ninth most common cause of death among all women in the US (and the fifth leading cause among non-Hispanic black women).

Worldwide the highest rate of nephritis are 50-55\% for African or Asian descent, then Hispanic at $43 \%$ and Caucasian at $17 \%$.

The average age of this inflammation (lupus nephritis in this case) is about 28.4 years old for an individual who has been diagnosed with the condition.

\section{Diagnosis}

- Urine test. A urinalysis might show red blood cells and red cell casts in your urine, an indicator of possible damage to the glomeruli. Urinalysis results might also show white blood cells, a common indicator of infection or inflammation, and increased protein, which can indicate nephron damage. Other indicators, such as increased blood levels of creatinine or urea, are red flags.

- Blood tests. These can provide information about kidney damage and impairment of the glomeruli by measuring levels of waste products, such as creatinine and blood urea nitrogen.

- Imaging tests. If your doctor detects evidence of damage, he or she may recommend diagnostic studies that allow visualization of your kidneys, such as a kidney X-ray, an ultrasound exam or a CT scan.

- Kidney biopsy. This procedure involves using a special needle to extract small pieces of kidney tissue for microscopic examination to help determine the cause of the inflammation. A kidney biopsy is almost always necessary to confirm a diagnosis of glomerulonephritis.

\section{Homoeopathic approach}

- Aconite: Suddenness is the hallmark of Aconite. Retention of urine in Newborns, from Fright; from cold, esp. Children, with crying and restlessness. Frequent urination during crisis. Fearful Restlessness. Oversensitive to noise, light, touch. Extreme excitability of nervous and vascular system. Desire open air.

- Apismellifica: Irritable. Quarrelsome. Shrieking with pain, in sleep. Loquacious delirium during fever. 
Stinging, Burning pains. Sudden, rapid developing complaints. Retention of Urine. Cystitis: burning pain. Shrieking from pain. Lower abdomen sensitive to touch.agg. HEAT; touch. Amel. Cold Application. Hot patient \&Thirstless.

- Belladonna: Reat intensity of symptoms. Senses acute. Sensitive to light, noise, jar. Irritability. Want to hit people, pull their hair, spit, bite. Knocks head against the wall. Violent impulses. Circulation: Congestion, flushes, Pulsating Pains. Pains (esp. Neuralgic) come and go suddenly. Convulsions, twitches SUDDEN onset. Violent attack. High fever. Burning heat; bright red discoloration. Throbbing pains, dryness. Hot head with cold extremities. Eyes glistening. Wild look. Go easily into delirium, hallucinations.

- Cantharis: Restlessness, fruitless. Restless mind. Fear of water, mirrors, shining objects (Stram.); death. Rage with crying, barking, howling. Frenzy. Violence (Stram.). Sudden stupor, unconsciousness. Burning PAINS. Violent pains, cutting, biting. Violent inflammation, rapid and intense. Amel. Lying. Amel. Warmth, but skin amel. Cold. Oversensitiveness of all parts. Cystitis/Urethritis violent BURNING pain, violent Tenesmus, intolerable urging, drops of urine, scalding, with restlessness, dances around the room in agony, screams from pain, urging, sitting amel., walking agg., pain agg. Drinking. Bloody urine. Nephritis: Pain in the back, sensitive to touch; colic.

- Equisetum: Cystitis: The Fuller The Bladder, The Less Discomfort. Less urging and pain when bladder is fuller. Resist the urging. Pain at end of urination (Nat-c, Sars). Constant desire to urinate without relief from passing great quantities of urine. Sensation of fullness after urination. Urine flows drop by drop. Incontinence with involuntary stools.Pain in region of right kidney extending to lower abdomen; with urging to urinate. Burning, cutting in urethra while urinating. Right lumbar region painful.

- Lycopodium: Timid. Fear of people. Counteracts. Boasts. Haughty. Egotism. Dictatorial towards those who can be controlled. Timid and passive towards superior. Bossy, dictatorial, rude at home. Timid at school; with strangers. Can also be generally timid, fearful. Sediment of red sand, brick-dust. Agg. 4 TO 8 P.M. (Hell), afternoon. Amel. Evening. Agg. Morning on waking. Amel. Open air.

- Pulsatilla: changeable symptoms. Warmblooded., agg. HEAT (but can be chilly), SUN, WARM ROOM, Getting feet wet, twilight.,amel. Open AIR, Cold Applications (Guai, Lac-c, Led, Sulph), Slow motion (Ferr). Wandering Pains (Form, Kali-bi, Kali-s). Recurrent cystitis. Urination involuntary, agg. Coughing, walking, sitting.

- Sarasaparilla: Dysuria in children. Child screams before and during urination. Urination frequent and copious. Unable to urinate during the day unless standing. Enuresis at night. Urine dribbles while sitting. Air passes from bladder during urination. Pain At End
Of Urination. Severe burning after urination. Pain from urethra back to abdomen. Pain from right kidney downward. Gravel or sediment in urine. Blood in urine.

- Terebinthina: Inflammation of kidneys or bladder with bloody, black or 'smokey' urine. Urine with coffee grounds. Urine with strong odor, like violets. Violent burning in whole urinary tract. Pain alternates between bladder and navel, amel. Walking in open air. Inflamed kidneys or bladder following any acute disease or after exanthema. Always associated with a varnished tongue.

\section{References}

1. Glomerulonephritis: Medline plus Medical Encyclopedia. www.nlm.nih.gov. Retrieved 2015-0614.

2. Interstitial nephritis: MedlinePlus Medical Encyclopedia. www.nlm.nih.gov. Retrieved 2015-0614.

3. American College of Rheumatology guidelines for screening, treatment, and management of lupus nephritis. National Guideline Clearinghouse. www.guideline.gov. Archived from the originalon 15 September 2016. Retrieved.

4. Keto Acids. Advances in Research and Application 2013. Reddy R, Sharma PK, Praveen Raj P. Homoeopathic treatment of intestinal. Research Journal of Recent Sciences ISSN, 2277, 2502. Edition 220e.

5. "Acute Nephritis; Nephrosis; Nephritic syndrome information. Patient | Patient. Patient. Retrieved 2016.

6. Pyelonephritis: Kidney Infection. www.niddk.nih.gov. Retrieved 2015-06-14.

7. Lupus Nephritis. www.niddk.nih.gov. Retrieved 201506-14."Nephritis Symptoms"esagil.org.

8. Shinton NK. Desk Reference for Hematology. CRC Press. ISBN 9781420005127. Retrieved 2019-02-14.

9. Glomerular Diseases. www.niddk.nih.gov. Retrieved 2015-06-15.

10. Oliguria: Background, Etiology, Epidemiology. Medscape. eMedicine. Retrieved 23 2016.

11. Uremia | accumulation in the blood of constituents normally eliminated in the urine that produces a severe toxic condition and usually occurs in severe kidney disease. www.merriam-webster.com. Retrieved 201506-14.

12. "Hematuria (Blood in the Urine)". www.niddk.nih.gov. Retrieved 2015-06-14.

13. Grabe M, Bartoletti R, Bjerklund Johansn TE, Cai T. Guidelines on Urological Infections. European Association of Urology; The Netherlands, 2015

14. Czaja CA, Scholes D, Hooton TM, Stamm WE. Population-based epidemiologic analysis of acute pyelonephritis. Clinical Infectious Diseases 2007;45(3):273-280.

15. Guarino N, Casamassima MG, Tadini B, Marras E, Lace R, Bianchi M. Natural history of vesicoureteral reflux associated with kidney anomalies. Urology 2005;65(6):1208-1211. 